\title{
Desarrollo de la lectura y la escritura de palabras con ortografía compleja: sus predictores
}

\section{Reading and Spelling of Orthographically Complex Words: Predictive Skills Desenvoluimento da leitura e a escritura de palauras com ortografia complexa: seus preditores}

\author{
Marina Ferroni*, Beatriz Diuk*, Milagros Mena* \\ Consejo Nacional de Investigaciones Cientificas y Técnicas (Conicet) y Centro de Investigaciones \\ Psicopedagógicas Aplicadas (CIPA), Universidad de San Martín, Argentina.
}

Doi: http://dx.doi.org/10.12804/apl34.2.2016.04

\section{Resumen}

Los objetivos de este trabajo fueron: (1) analizar la interacción entre los procesos fonológicos y la adquisición del conocimiento ortográfico en niños que aprenden a leer y escribir en español y (2) analizar qué habilidades predicen el aprendizaje ortográfico. Para ello se realizó un estudio longitudinal de primer a tercer grados con 46 sujetos hispanohablantes. A principio de primer grado se administraron pruebas para medir las habilidades predictoras de conocimiento ortográfico. A finales de los grados primero a tercero se administró una prueba de lectura y escritura de palabras ortográficamente complejas y de seudopalabras. Los resultados mostraron, en concordancia con investigaciones pasadas, que en momentos iniciales los niños leen y escriben palabras accediendo al procesamiento fonológico, y que a partir de segundo grado el conocimiento ortográfico empieza a incrementarse y a tener como mejores predictores las habilidades de denominación rápida y fluidez en el trazado de las letras.

Palabras clave: lectura; escritura; ortografía; fonología; predictores.

\section{Abstract}

This paper analyzed the interaction between phonological processes and the acquisition of orthographic knowledge in children learning to read and spell in Spanish. The study explored which abilities predicted the orthographic knowledge involved in the recognition and spelling of words including inconsistent correspondences. A longitudinal study was conducted with 461 st to $3 \mathrm{rd}$ grade children. At the beginning of 1 st grade children were given tests evaluating abilities that are considered potential predictors of orthographic knowledge acquisition. At the end of 1st, 2nd and 3rd grades tests of reading and spelling of orthographically

* Son miembros del Consejo Nacional de Investigaciones Científicas y Técnicas (Conicet) y del Centro de Investigaciones Psicopedagógicas Aplicadas (CIPA), Universidad de San Martín, Argentina.

La correspondencia relativa a este artículo debe ser enviada a Marina Ferroni, CIPA, Universidad de San Martín, Martín de Irigoyen 3100 (1650), San Martín, Buenos Aires, Argentina. Correo electrónico: ferronimarina@gmail.com

Cómo citar este artículo: Ferroni, M., Diuk, B. \& Mena, M. (2016). Desarrollo de la lectura y la escritura de palabras con ortografía compleja: sus predictores. Avances en Psicología Latinoamericana, 34(2), 253-271. doi: http://dx.doi.org/10.12804/ apl34.2.2016.04 
complex words as well as of pseudowords were administered. Results from the reading and spelling tests showed that at the beginning children read and spell through phonological processing and that starting 2 nd grade orthographic knowledge increases. This knowledge was predicted by rapid naming abilities as well as fluency in letter writing.

Keywords: reading; writing; spelling; phonology; predictors

\section{Resumo}

O objetivo deste trabalho foi analisar a interação entre os processos fonológicos e a aquisição do conhecimento ortográfico em crianças que aprendam a ler e a escrever em espanhol. $\mathrm{O}$ estudo também se propôs analisar que habilidades predizem a aprendizagem ortográfica refletido na velocidade no reconhecimento de palavras e na escritura convencional de palavras com correspondências inconsistentes e dependentes do contexto. Para isto, realizou-se um estudo longitudinal de 1er a 3er ano com 46 sujeitos hispanofalantes. No começo do primeiro ano se administraram provas para medir as habilidades preditores de conhecimento ortográfico. No fim de 1ro, 2do y 3ro ano se administrou a prova de leitura e escritura de palavras ortograficamente complexas e de pseudopalavras. Os resultados das provas de leitura e escritura mostraram, em concordância com pesquisas passadas, que em momentos iniciais as crianças leem e escrevem palavras acedendo ao processamento fonológico, e que a partir do 2 do ano o conhecimento ortográfico começa a incrementar-se tendo como melhores preditores às habilidades de denominação rápida e fluidez no traço das letras.

Palavras-chave: leitura; escritura; ortografia; fonologia; preditores.

Los estudios sobre el desarrollo de la lectura y la escritura en el marco de la psicología cognitiva han señalado una importante interacción entre los procesos fonológicos y ortográficos durante el aprendizaje (Ehri, 1992; Perfetti, 1992; Share, 2011). En términos generales, estos modelos plantean que el procesamiento fonológico de las palabras permite incrementar el conocimiento sobre la forma ortográfica de los ítems léxicos (Perfetti, 1992).

Ahora bien, la mayoría de los estudios que han abordado la interacción fonológica-ortografía se han llevado a cabo en lenguas de ortografías opacas en las cuales la relación entre la fonología y la ortografía resulta muy irregular. David Share (1995, 2011), a partir de estudios realizados en hebreo, una lengua de ortografía transparente como el español, en la cual la relación entre fonología y ortografía resulta mucho más estable que en inglés, planteó la hipótesis de la recodificación fonológica como mecanismo autodidacta. Sostiene esta hipótesis que el mecanismo de recodificación fonológica, por el cual los grafemas son convertidos en fonemas por aplicación de reglas de conversión y los fonemas son ensamblados para su pronunciación (Ferreres, Martínez, Jacubovich, Olmedo \& López, 2003; Signorini \& Piacente, 2001), opera como un mecanismo de aprendizaje de la forma ortográfica de las palabras, ya que el reconocer una palabra por la vía fonológica promueve el almacenamiento de su representación ortográfica.

Sostiene Share (2004) que los lectores principiantes de lenguas de ortografía transparente, al ser capaces de leer y escribir basándose en el procesamiento fonológico, serían relativamente insensibles a las características ortográficas de las palabras y sugiere que se requeriría una cantidad considerable de exposición a la escritura para poder comenzar a adquirir conocimiento ortográfico. Los estudios realizados por dicho autor (Share, 2004) señalaron que en una lengua de ortografía transparente el almacén léxico comenzaría a incrementarse significativamente a mediados del segundo año escolar.

En coincidencia, un estudio realizado con niños argentinos, Sánchez, Diuk, Borzone y Ferroni (2009) administraron una prueba de escritura de palabras que permitían analizar diferentes variables (complejidad fonológica y ortográfica, extensión y frecuencia de las palabras) a niños de los grados 
primero y segundo. Los resultados mostraron que los mecanismos fonológicos son fundamentales en la primera etapa de aprendizaje de la escritura. Al finalizar el segundo año escolar, se observó también una interacción entre los mecanismos fonológicos y léxicos en español.

En efecto, el español es una lengua de ortografía transparente, en la cual la mayor parte de las relaciones grafema-fonema son simples, es decir, generalmente a la misma letra corresponde siempre el mismo sonido y viceversa (como en el caso de las vocales). Pero a pesar de la transparencia de la ortografía, existe un número limitado de irregularidades en las correspondencias que complejizan las tareas de lectura y escritura.

Efectivamente, además de las correspondencias simples (unívocas), existen correspondencias que dependen del contexto silábico (correspondencias dependientes del contexto). Por ejemplo, en la lectura, si el grafema $G$ precede a $A, O$ y $U$ se pronuncia /g/; pero si precede a $E$ o $I$ se pronuncia $/ \mathrm{x}$ / habiendo una regla fija que específica la pronunciación en cada caso. El conocimiento de las correspondencias grafema-fonema simples y dependientes del contexto permite la lectura de cualquier palabra del idioma.

En la dirección de fonemas a grafemas, las relaciones resultan más complejas (Jiménez etal., 2008; Signorini, Borzone \& Diuk, 2001), ya que existen en el proceso de escritura, además de las correspondencias simples y dependientes del contexto, correspondencias inconsistentes. Las inconsistencias en las correspondencias se dan cuando a un fonema corresponden dos o más grafemas, sin que exista ninguna regla que especifique cuál debe utilizarse en cada caso (por ejemplo, en la palabra viento no hay ninguna regla que especifique si la palabra se escribe con $V$ o con $B$ ).

Es posible pensar que las características del sistema ortográfico del español generen importantes diferencias en las estrategias de los niños para la lectura o la escritura de palabras con ortografía compleja. En efecto, mientras que la lectura en español puede resolverse a partir del procesamiento fonológico y el conocimiento de reglas de correspondencia, para la escritura convencional de palabras con correspondencias inconsistentes resulta necesario poseer representaciones ortográficas de las palabras (Perfetti, 1992). Por ello, la escritura convencional de palabras con este tipo de correspondencias, así como el reconocimiento veloz de las palabras en la lectura, resultan indicadores de que los sujetos han adquirido conocimiento léxico. Resultan, sin embargo, escasos los trabajos que centran su análisis en la escritura de palabras con correspondencias inconsistentes o en medidas de velocidad lectora en español.

El presente estudio se propone: (1) analizar longitudinalmente la interacción entre procesos fonológicos y ortográficos en el aprendizaje de la lectura y la escritura por parte de niños hablantes de español. Y (2) analizar cuáles son las habilidades que predicen la escritura ortográficamente convencional de palabras con correspondencias inconsistentes o la velocidad en la lectura de palabras en español.

Dada la transparencia del sistema ortográfico, se ha obtenido evidencia de la importancia de las habilidades de conciencia fonológica — habilidad para reconocer y manipular los sonidos del habla (Mattingly, 1972) - y el conocimiento sobre las correspondencias entre letras y sonidos de los sujetos (Cardoso-Martins \& Corrêa, 2008; Salles \& Parente, 2006; Share, 1995) como precursores de la lectura y la escritura en momentos iniciales del desarrollo. Sin embargo, a pesar de reconocer su importancia en momentos iniciales, se ha señalado que el rol predictivo de la conciencia fonológica sobre la lectura tendría una importancia temporalmente limitada. En efecto, diferentes estudios señalan que, una vez se han alcanzado altos niveles de precisión en la lectura de palabras, serían las tareas de denominación rápida las que predicen la velocidad lectora, es decir, el tiempo que demora un sujeto en reconocer una palabra (McCrory, Meche1li, Frith \& Price, 2005; Wimmer, 2006; Wimmer 
\& Mayringer, 2002). Las habilidades de denominación rápida permitirían medir la velocidad con la cual los sujetos acceden a una etiqueta fonológica a partir de un símbolo gráfico (Manis, Seidenberg \& Doi, 1999; Savage, Pillay \& Melidona, 2008).

Asimismo, existe en la literatura sobre el tema una línea de desarrollo que sitúa los procesos de atención visual como importantes predictores del conocimiento ortográfico. Estos estudios señalan la importancia de las habilidades atencionales hacia el material gráfico, que se complementarían con los procesos fonológicos para permitir el almacenamiento de representaciones ortográficas en el léxico mental (Bosse, Tanturier \& Valdois, 2007; Bourke \& Adams, 2010; Plaza \& Cohen, 2006).

Por último, se ha hecho referencia a la importancia de los procesos motores en el desarrollo de la escritura (Berninger et al., 1992; Shahar-Yames \& Share, 2008; Treiman \& Bourassa, 2000). En este sentido, se ha sugerido que los movimientos realizados al trazar las formas y la secuencia de las letras de las palabras (más allá de la calidad caligráfica del output) pueden originar la formación de representaciones de patrones grafomotores que generarían un link adicional entre las formas fonológicas y las formas ortográficas (Berninger et al., 1992; Cunningham \& Stanovich, 1991). Resulta interesante preguntarse si las habilidades relacionadas con los procesos motores, como la fluidez en el trazado de las letras, se relacionan con la formación de representaciones ortográficas de las palabras en español.

En síntesis, el objetivo de este trabajo es analizar la interrelación entre el procesamiento fonológico y ortográfico durante el aprendizaje de la lectura y la escritura, así como analizar las habilidades que predicen el desarrollo de conocimiento ortográfico que se refleja en la escritura convencional de palabras con correspondencias inconsistentes y en la velocidad lectora. Para ello se realizó un estudio longitudinal en el cual se evaluó un grupo de niños a lo largo de los tres primeros años de escolarización.

\section{Método}

Se trata de un diseño longitudinal de grupo (Hernández, Fernández \& Baptista, 2006) en el cual se examinan cambios en el tiempo respecto de alguna variable en un grupo específico.

\section{Participantes}

Participaron de este estudio 46 sujetos ( 22 niños y 24 niñas) que asistían a un colegio privado que atiende a una población de clase media-alta de la provincia de Buenos Aires, Argentina.

Con anterioridad al comienzo de las sesiones de evaluación, las familias de los niños firmaron un consentimiento informado en el que expresaban su conformidad en relación con el proyecto de investigación. Los niños participantes manifestaron su asentimiento de forma verbal y en todo momento se atendió a que no se produjera ningún tipo de malestar que justificara la exclusión de algún niño de las sesiones de aprendizaje. Todos los participantes eran hispanohablantes nativos.

De la muestra inicial, se separó a una niña que presentaba dificultades de aprendizaje según señalaron profesionales de la institución. La escuela informó que el resto de los niños no presentaba problema neuropsicológico alguno que afectara el normal desempeño escolar.

\section{Materiales}

\section{Principio de primer grado: tiempo 1 (T1)}

Conciencia fonológica: se administraron tres subtests de la batería elaborada por Jiménez y Ortiz (1995) con el fin de conocer el nivel de conciencia fonológica de cada niño o niña:

- Prueba de reconocimiento de sonido inicial (estadístico de alfa de Cronbach de .63). Esta prueba constaba de 5 ítems en los cuales los niños debían reconocer el sonido inicial de palabras 
que comenzaban con los grafemas $A, S, D, P$ y $S$. En esta prueba las evaluadoras mostraban una lámina con 4 dibujos de elementos cuyos nombres comenzaban con diferentes sonidos. Luego se les pedía a los niños que señalaran qué dibujo tenía un nombre que comenzaba con X sonido.

- Prueba de síntesis de sonidos (estadístico de alfa de Cronbach de .94). La prueba de síntesis de sonidos constó de 10 palabras de 1 y 2 sílabas de estructura fonológica simple (es decir, estructura consonante-vocal; solo se incluyó una palabra con grupo consonántico: flan). En esta prueba, las evaluadoras iban pronunciando los sonidos de cada palabra y los niños debían sintetizar los sonidos para arribar a la pronunciación de las palabras.

- Prueba de omisión de sílabas inicial y final (estadístico de alfa de Cronbach de .81). Esta prueba constaba de 14 palabras de 2 y 3 sílabas de estructura fonológica simple, presentadas de manera oral a los niños para que omitieran en 7 de ellas la sílaba inicial y en 7, la sílaba final. En esta prueba, las evaluadoras les decían a los niños, por ejemplo: "si a la palabra auto le saco la primera parte, me queda to, y si le saco la primera parte a mono, ¿qué me queda?".

- Atención visual: se administró una prueba de atención visual adaptada de Plaza y Cohen (2006) con el objetivo de analizar la velocidad y precisión con la cual los niños eran capaces de reconocer un determinado input visual (véase anexo). Se presentaba a los niños una forma gráfica y luego una matriz compuesta por dicha forma repetida 10 veces e intercalada entre otros estímulos similares. Los niños debían marcar en la matriz impresa todos los target que pudiera identificar en un minuto.

Fluidez en el trazado de letras: se solicitó a los niños que copiaran en el menor tiempo posible una oración que contiene el $55 \%$ de las letras del alfabeto. Se trata de una tarea elaborada por
Berninger y Graham (1997) y adaptada al español por Sánchez, Borzone y Diuk (2007). La oración que se debía copiar era: El puma bravo y feroz corre a la gallina hasta el jardín. En esta prueba se contabilizaba la cantidad de letras que los niños eran capaces de copiar en un minuto.

Conocimiento de letras: se presentaron a los niños 25 letras impresas en imprenta mayúscula $y$ en fuente Arial tamaño 72. Las letras fueron expuestas de una en una ante cada niño. A continuación, se les preguntaba a los sujetos si conocían el nombre. Luego, sin importar si la respuesta había sido correcta o incorrecta, se les preguntaba si conocían el sonido de esta. Se puntuó por separado el conocimiento del nombre y el conocimiento del sonido de las letras, asignando un punto por cada respuesta correcta. El estadístico de alfa de Cronbach para la identificación del nombre de la letra fue de .86; mientras que para la producción del sonido de las letras fue de .92 .

Dictado de letras por sonidos: en esta prueba la evaluadora (primera autora de este trabajo) pronunciaba una serie de sonidos (por ejemplo, la evaluadora decía $m m m$...) y los niños debían escribir el grafema correspondiente. El estadístico de alfa de Cronbach fue de .85 .

Denominación rápida: se evaluó al grupo mediante tres pruebas de denominación rápida. Dos de ellas estuvieron compuestas por matrices con letras: la prueba tradicional de RAN (Denckla \& Rudell, 1976) y una prueba alternativa de RAN de letras adaptada de Compton (2003) para este trabajo. El objetivo de esta prueba alternativa fue agregar complejidad al procesamiento fonológico de la tarea tradicional. Para ello, la matriz de la prueba de denominación rápida tradicional (en la cual se encuentran las letras $o, a, s, d$ y $p$ ) se modificó omitiendo uno de los ítems (o) y agregando otro $(t)$ que, en español, rima con dos letras del resto de la matriz $(p$ y $d)$. La tercera fue una prueba 
de denominación rápida de dígitos. En esta prueba se presenta una matriz de símbolos gráficos distribuidos en 5 columnas por 6 filas que los sujetos evaluados deben nombrar en el menor tiempo posible.

Fin de primer grado: tiempo 2 (T2), fin de segundo grado: tiempo 3 (T3) y fin de tercer grado: tiempo 4 (T4)

Escritura y lectura de palabras ortográficamente complejas: se administró una prueba de lectura y escritura de 93 palabras ortográficamente complejas, 74 de las cuales incluían correspondencias no unívocas. Entre ellas se incluyeron grafemas compuestos por más de una letra (como $G U$, $C H, L L$ y $Q U$ ), correspondencias inconsistentes dominantes (es decir, de mayor frecuencia en el sistema ortográfico del español como $B$ ) y no dominantes (es decir, de menor frecuencia en el sistema ortográfico del español como $V$ ) y correspondencias dependientes ( $G U$ antes de $E$ o $I$ ) e independientes del contexto $(\mathrm{CH})$. Las palabras seleccionadas fueron de alta y baja frecuencia según el Diccionario de frecuencias del castellano escrito de niños de 6 a 12 años (Martínez \& García, 2004). Las mismas palabras se presentaron para la lectura y se dictaron para su escritura, y así se contrabalanceó el orden de presentación. En la lectura, se asignó un punto a cada palabra leída de manera correcta y se midió el tiempo de lectura.

En la prueba de escritura de palabras se puntuó por separado la correcta escritura de correspondencias inconsistentes y dependientes del contexto a fin de realizar los análisis de datos posteriores con ambos tipos de dificultades disociadas. En las medias obtenidas en la escritura, el estadístico de alfa de Cronbach fue de .92 en la escritura de correspondencias dependientes del contexto, y de .90 en la escritura de correspondencias inconsistentes a fin de primer grado, de .80 en la escritura de correspondencias dependientes del contexto, de .82 en la escritura de correspondencias inconsistentes a fin de segundo grado y de .87 en la escritura de correspondencias dependientes del contexto y de .90 en la escritura de correspondencias inconsistentes a fin de tercer grado. En el caso de la lectura, el estadístico de alfa de Cronbach fue de .95 a fin de primer grado, de .98 a fin de segundo grado y .85 a fin de tercero.

Lectura y escritura de seudopalabras: se administró la prueba de lectura de seudopalabras del test LEE de Lectura y Escritura en Español (Defior etal., 2006). Se trata de una prueba de lectura y escritura de 32 seudopalabras, es decir, de cadenas de letras inventadas, cuya combinación respeta las regularidades del sistema fonológico del español. Las seudopalabras podían tener 1, 2, 3, 4 o 5 sílabas de estructura simple y compleja y contenían correspondencias simples y dependientes del contexto en la lectura y correspondencias simples, dependientes del contexto e inconsistentes en la escritura. Se asignó un punto a cada seudopalabra leída de manera correcta y se midió el tiempo de lectura en el T3 y T4. Para la lectura, el estadístico de alfa de Cronbach de esta prueba fue de .96 a fin de primer grado, de .64 a fin de segundo grado y de .68 a fin de tercero. Para la escritura, el estadístico de alfa de Cronbach fue .87 a fin de primer grado, de .85 a fin de segundo grado y de .66 a fin de tercero.

\section{Procedimiento}

Las autoras de este trabajo evaluaron a los niños a principio de primer grado y a fin de primero, segundo y tercer grados. La prueba de dictado de sonidos constituyó una excepción, ya que fue administrada solo por la primera autora del trabajo.

Las pruebas de escritura de palabras y seudopalabras fueron administradas a fin de los grados primero, segundo y tercero, de manera grupal, en el aula de clase de los niños. Todas las pruebas restantes se administraron de manera individual 
en un aula de la escuela, en la cual no se estaba desarrollando ninguna otra actividad.

Se llevó a cabo una sesión individual de 20 minutos aproximadamente a fin de los grados primero, segundo y tercero, en la cual se evaluó exclusivamente la lectura de palabras y de seudopalabras.

Para evaluar las habilidades predictoras de la adquisición de conocimiento ortográfico en el T1 se realizaron 2 sesiones individuales de evaluación de aproximadamente 20 minutos cada una: en una sesión se incluyeron las pruebas de denominación rápida de letras y números, la prueba de omisión de sílabas, la prueba de síntesis de sonidos y el reconocimiento de las letras por sus sonidos. En la sesión 2 se administraban la prueba de reconocimiento de sonido inicial, la prueba de reconocimiento del nombre y el sonido de las letras, la prueba de denominación rápida alternativa y la prueba de atención visual.

\section{Resultados}

En primer lugar, se inspeccionaron las distribuciones de los puntajes obtenidos en los cuatro tiempos de evaluación. Dicho análisis permitió observar que, a principio de primer grado, las medidas que se alejaron significativamente de la distribución normal asintótica fueron las pruebas de síntesis de sonidos ( $Z$ de Kolmogorov-Smirnov $=1.650 ; p=.009)$, la prueba de reconocimiento de sonido inicial $(Z$ de Kolmogorov-Smirnov $=$ $2.512 ; p=.000)$ y la lectura de seudopalabras $(Z$ de Kolmogorov-Smirnov $=2.001 ; p=.001)$. Por su parte, a fin de primer grado, las tareas que se alejaron significativamente de la distribución normal asintótica fueron las pruebas de síntesis de sonidos $(Z$ de Kolmogorov-Smirnov = 1.721; $p=.004)$, reconocimiento de sonido inicial ( $Z$ de Kolmogorov-Smirnov $=3.857 ; p=.001$ ), lectura de seudopalabras $(Z$ de Kolmogorov-Smirnov $=$ $1.594 ; p=.012)$ y la escritura de seudopalabras $(Z$ de Kolmogorov-Smirnov $=1.574 ; p=.014)$; entre tanto, a fin de segundo grado las tareas que se alejaron significativamente de la distribución normal asintótica fueron la prueba de lectura de palabras ortográficamente complejas ( $Z$ de Kolmogorov-Smirnov $=1.622 ; p=.010)$ y la medida de tiempo de lectura de palabras ortográficamente complejas y ( $Z$ de Kolmogorov-Smirnov = 1.444; $p=.031)$. Para corregir la falta de normalidad se transformaron las puntuaciones a su logaritmo natural; sin embargo, al no ser posible realizar dicha corrección, se utilizaron para el análisis estadístico con dichas medidas pruebas no paramétricas. Por último, el análisis de las medidas obtenidas a fin de tercer grado mostró que la totalidad de las pruebas tuvieron una distribución normal. En segundo lugar, se calcularon los estadísticos descriptivos de todas las medidas evaluadas, los cuales se expresan en porcentajes en la tabla 1 .

La inspección de los resultados mostró un desempeño bajo a fin de primer grado en la escritura de palabras con correspondencias dependientes del contexto ( $42 \%$ ) e inconsistentes $(41 \%)$, lo que arroja evidencia de un bajo nivel de conocimiento ortográfico por parte de los sujetos en este momento del desarrollo. En efecto, las medias obtenidas en escritura señalan la primacía del uso de recursos fonológicos en esta tarea. El desempeño mejoró lentamente en los tiempos de evaluación posteriores $(62 \%$ en las palabras con correspondencias dependientes del contexto y $58 \%$ en las inconsistentes a fin de segundo, y $76 \%$ en las palabras con correspondencias dependientes del contexto y $64 \%$ en las inconsistentes a fin de tercero). Dado que las medias de la escritura ortográficamente convencional de fin de primer grado estuvieron por debajo del nivel del azar, no se consideraron en los análisis subsiguientes como medidas de aprendizaje ortográfico.

En la lectura de palabras, el desempeño en precisión fue alto a fines de primer grado $(75 \%) \mathrm{y}$ siguió incrementándose a fines de segundo (94\%) y tercer grados $(95 \%)$. 
Tabla 1

Estadísticos descriptivos correspondientes a las habilidades evaluadas a principio de primer grado y a fin de los grados primero, segundo y tercero (expresados en porcentajes)

\begin{tabular}{|c|c|c|c|c|c|c|c|c|}
\hline & \multicolumn{2}{|c|}{ Ppio. $1^{\text {er }}$ grado } & \multicolumn{2}{|c|}{ Fin $1^{\mathrm{er}}$ grado } & \multicolumn{2}{|c|}{ Fin $2^{\text {do }}$ grado } & \multicolumn{2}{|c|}{ Fin $3^{\text {er }}$ grado } \\
\hline & M & $\mathrm{DE}$ & M & $\mathrm{DE}$ & M & $\mathrm{DE}$ & M & $\mathrm{DE}$ \\
\hline Atención visual & 80.8 & 25.1 & - & - & - & - & - & - \\
\hline Conciencia fonológica & & & - & - & & & & \\
\hline Omisión de sílabas & 41.4 & 25.4 & - & - & - & - & - & - \\
\hline Síntesis de sonidos & 36.9 & 39.4 & - & - & - & - & - & - \\
\hline Rec. sonido inicial & 84.3 & 25.6 & - & - & - & - & - & - \\
\hline \multicolumn{9}{|l|}{ Denominación rápida } \\
\hline Letras (dif. fonológica) & 54.8 & 18.1 & - & - & - & - & - & - \\
\hline Letras & 58.1 & 22.1 & - & - & - & - & - & - \\
\hline Dígitos & 48.9 & 13.0 & & & - & - & - & - \\
\hline \multicolumn{9}{|l|}{ Conocimiento de letras } \\
\hline Nombre de letras & 87.0 & 12.0 & - & - & - & - & - & - \\
\hline Sonido de letras & 66.9 & 25.2 & - & - & - & - & - & - \\
\hline Dictado de sonidos & 84.1 & 18.0 & - & - & - & - & - & - \\
\hline Fluidez en el trazado & 50.9 & 12.5 & - & - & - & - & - & - \\
\hline \multicolumn{9}{|l|}{ Lectura } \\
\hline Seudopalabras & - & - & 77.6 & 23.3 & 88.1 & 12.50 & 88.90 & 10.80 \\
\hline Seudopalabras por segundo & - & - & - & - & 0.23 & .04 & 0.44 & .72 \\
\hline Palabras & - & - & 75.4 & 22.5 & 93.5 & 5.90 & 94.90 & 5.50 \\
\hline Palabras por segundo & - & - & - & - & 1.02 & .54 & 1.06 & .03 \\
\hline \multicolumn{9}{|l|}{ Escritura } \\
\hline Seudopalabras & - & - & 64.6 & 20.4 & 67.1 & 21.00 & 86.20 & 10.00 \\
\hline Palabras (corres. inc.) & - & - & 40.6 & 19.8 & 58.3 & 14.50 & 64.80 & 14.70 \\
\hline Palabras (corres. dep.) & & & 41.6 & 21.0 & 62.0 & 21.30 & 76.50 & 16.70 \\
\hline
\end{tabular}

$\mathrm{M}=$ media; $\mathrm{DE}=$ desvío estándar.

\section{Comparaciones entre medidas obtenidas}

La comparación entre lectura y escritura mostró que el grupo de sujetos tuvo un mejor desempeño en la lectura que en la escritura de palabras con ortografía compleja en los tres tiempos de evaluación. Con el objetivo de evaluar el grado de significatividad de las diferencias encontradas entre las medias de lectura y escritura de fines de los grados primero a tercero, se utilizó una prueba no paramétrica de contraste (U de Wilcoxon) para las medidas de fin de los grados primero y segundo, y una prueba de $t$ para muestras relacionadas para las medidas de fin de tercer grado. El análisis mostró en todos los grados diferencias estadísticamente significativas entre las pruebas, a favor de la lectura: $Z=5.4$, 
$p=.001$ a fin de primer grado, y en comparación a la escritura de correspondencias dependientes del contexto; $Z=5.86, p=.001$ a fin de primer grado y en comparación a la escritura de correspondencias inconsistentes; $Z=5.93, p=.001$ a fin de segundo grado y en comparación a la escritura de correspondencias dependientes del contexto; $Z=5.83$, $p=.001$ a fin de segundo grado y en comparación a la escritura de correspondencias inconsistentes, y $t(45)=10.7, p=.001$, a fin de tercer grado y en comparación a la escritura de correspondencias dependientes del contexto y $t(45)=13.2, p=.001$, a fin de tercer grado y en comparación a la escritura de correspondencias inconsistentes.

Por otra parte, la comparación entre la escritura de palabras y seudopalabras mostró en todos los grados diferencias estadísticamente significativas a favor de la escritura de seudopalabras: $Z=3.2$, $p=.001$ a fin de primer grado y en comparación a la escritura de correspondencias dependientes del contexto; $Z=3.86, p=.001$ a fin de primer grado y en comparación a la escritura de correspondencias inconsistentes; $Z=4.43, p=.001$ a fin de segundo grados y en comparación a la escritura de correspondencias dependientes del contexto; $Z=4.73$, $p=.001$ a fin de segundo grado y en comparación a la escritura de correspondencias inconsistentes; $Z=5.20, p=.001$ a fin de tercer grado y en comparación a la escritura de correspondencias dependientes del contexto, y $Z=5.90, p=.001$ a fin de tercer grado y en comparación a la escritura de correspondencias inconsistentes.

Con respecto a los tiempos de lectura, los resultados dejaron ver que, a fin de segundo grado, los sujetos leyeron aproximadamente una palabra por segundo; mientras que necesitaron 5 segundos para recodificar una seudopalabra. Por su parte, en tercer grado el tiempo de lectura se redujo a 1.6 palabras por segundo; mientras que la lectura de seudopalabras se redujo a 2.5 segundos por seudopalabra. La diferencia entre el tiempo de lectura de palabras y seudopalabras fue significativa en ambos grados a favor de la lectura de palabras $(t[45]=-537.8, p=.001$ en segundo y $t[45]=$ $-90.75, p=.001$ en tercer grado). Por otro lado, la comparación del tiempo de lectura entre grados señaló la existencia de diferencias significativas a favor de la lectura de tercer grado en comparación al desempeño en segundo grado $(t[45]=8.51$, $p=.001$ para las palabras y $t[45]=3.58, p=.001$ para las seudopalabras).

El análisis de los datos también reveló un efecto de frecuencia, ya que en todos los momentos de evaluación los niños escribieron y leyeron significativamente mejor las palabras frecuentes que las poco frecuentes $(Z=3.71, p=.001$, para la lectura de fin de primer grado, $t[45]=17.5, p=.001$, para la escritura de principio de segundo grado, y $Z=4.99, p=.001$ para la lectura de fin de segundo grado, y $Z=5.19, p=.001$, para la lectura de fin de tercer grado).

Por último, la comparación de las medidas de escritura de correspondencias dependientes del contexto e inconsistentes señaló que no existieron diferencias significativas en el desempeño entre ambas a fin de primer grado $(t[39]=-.792$, $p=.433)$ ni a fin de segundo grado $(t[45]=-1.16$, $p=.110)$. El análisis de los datos reveló la existencia de diferencias significativas a fin de tercer grado a favor de la escritura de correspondencias dependientes del contexto $(t[45]=-6.45, p=.001)$ en comparación con la escritura de correspondencias inconsistentes. Estos datos sugieren que la adquisición de reglas contextuales sigue un patrón bastante similar a la adquisición de información léxica que se diferencia de la formación de representaciones ortográficas recién a fines de tercer grado.

\section{Correlaciones}

Con el fin de analizar las relaciones entre diferentes tareas (lectura y escritura de palabras y seudopalabras) entre sí, y de intentar inferir el tipo de procesamiento al que se accede en cada caso (fonológico a través de la lectura y escritura de seudopalabras y ortográfico a través de la lectura y 


\section{Marina Ferroni, Beatriz Diuk, milagros mena}

escritura de palabras ortográficamente complejas), hecho que resulta de importancia para el análisis del desarrollo de la escritura y la lectura de palabras ortográficamente complejas, se calcularon correlaciones bivariadas (Rho de Spearman, dado que se incluyen medias que se alejaron significativamente de la distribución normal asintótica) entre las distintas pruebas de lectura y escritura administradas (véase tabla 2).

Los resultados mostraron correlaciones de moderadas $(+.40 \mathrm{a}+.60)$ a altas $(+.60 \mathrm{a}+.80$, según Maisto \& Morris, 2001) entre todas las medidas de

Tabla 2

Correlaciones entre las medidas de lectura y escritura de palabras con correspondencias inconsistentes (debajo de la diagonal)-correspondencias dependientes del contexto (arriba de la diagonal) y seudopalabras de los tres tiempos de evaluación

\begin{tabular}{|c|c|c|c|c|c|c|c|c|c|c|c|c|c|c|c|c|}
\hline & 1 & 2 & 3 & 4 & 5 & 6 & 7 & 8 & 9 & 10 & 11 & 12 & 13 & 14 & 15 & 16 \\
\hline $\begin{array}{l}\text { 1-Escritura de pala- } \\
\text { bras (T2) }\end{array}$ & 1.000 & .073 & $.706^{* *}$ & .067 & $.606^{* *}$ & -.012 & -.177 & .289 & .132 & -.155 & .023 & .014 & -.181 & .131 & .016 & .016 \\
\hline $\begin{array}{l}\text { 2-Lectura de palabras } \\
\text { (T2) }\end{array}$ & -.104 & 1.000 & $.739^{* *}$ & $.770^{* * *}$ & -.107 & $.830^{* * *}$ & $-.456^{* *}$ & $.557^{* *}$ & $.694^{* *}$ & $-.592^{* *}$ & $.422^{* *}$ & $.775^{* *}$ & $-.576^{* *}$ & $.601^{* *}$ & $.756^{* *}$ & $.756^{* *}$ \\
\hline $\begin{array}{l}\text { 3-Escritura de seudo- } \\
\text { palabras (T2) }\end{array}$ & .068 & $.739^{* *}$ & 1.000 & $.658^{* * *}$ & .051 & $.623^{* *}$ & $-.390^{* *}$ & $.610^{* *}$ & $.588^{* *}$ & $-.429^{* * *}$ & $.490^{* * *}$ & $.531^{* * *}$ & $-.401^{* *}$ & $.577^{* *}$ & $.478^{* * *}$ & $.478^{* * *}$ \\
\hline $\begin{array}{l}\text { 4-Lectura de seudo- } \\
\text { palabras (T2) }\end{array}$ & -.104 & $.770^{* *}$ & $.658^{* *}$ & 1.000 & -.166 & $.630^{* *}$ & $-.389^{* *}$ & $.482^{* *}$ & $.595^{* *}$ & $-.516^{* *}$ & $.472^{* *}$ & $.708^{* *}$ & $-.504^{* *}$ & $.565^{* *}$ & $.680^{* * *}$ & $.680^{* *}$ \\
\hline $\begin{array}{l}\text { 5-Escritura de pala- } \\
\text { bras (T3) }\end{array}$ & .254 & .063 & .051 & -.040 & 1.000 & -.061 & .038 & .079 & .119 & .067 & .154 & -.012 & .027 & .064 & -.129 & -.129 \\
\hline $\begin{array}{l}\text { 6-Lectura de palabras } \\
\text { (T3) }\end{array}$ & -.155 & $.830^{* *}$ & $.623^{* * *}$ & $.630^{* *}$ & .004 & 1.000 & $-.430^{* * *}$ & $.453^{* * *}$ & $.686^{* *}$ & $-.549^{* *}$ & $.463^{* *}$ & $.780^{* * *}$ & $-.528^{* *}$ & $.516^{* *}$ & $.705^{* * *}$ & $.705^{* * *}$ \\
\hline $\begin{array}{l}\text { 7-Tiempo de lectura } \\
\text { de palabras (T3) }\end{array}$ & -.112 & $-.456^{* *}$ & $-.390^{* *}$ & $-.389^{* * *}$ & -.032 & $-.430^{* *}$ & 1.000 & -.150 & $-.294^{*}$ & $.701^{* *}$ & $-.374^{*}$ & $-.466^{* * *}$ & $.566^{* *}$ & -.239 & $-.443^{* *}$ & $-.443^{* *}$ \\
\hline $\begin{array}{l}\text { 8-Escritura de seudo- } \\
\text { palabras (T3) }\end{array}$ & .083 & $.557^{* *}$ & $.610^{* * *}$ & $.482^{* *}$ & .283 & $.453^{* * *}$ & -.150 & 1.000 & $.495^{* *}$ & $-.312^{*}$ & $.357^{*}$ & $.486^{* *}$ & -.246 & $.451^{* *}$ & $.527^{* * *}$ & $.527^{* * *}$ \\
\hline $\begin{array}{l}\text { 9-Lectura de seudo- } \\
\text { palabras (T3) }\end{array}$ & -.168 & $.694^{* *}$ & $.588^{* *}$ & $.595^{* *}$ & .216 & $.686^{* *}$ & $-.294^{*}$ & $.495^{\text {** }}$ & 1.000 & $-.527^{* *}$ & $.453^{* *}$ & $.716^{* * *}$ & $-.578^{* *}$ & $.516^{* *}$ & $.567^{\text {*** }}$ & $.567^{* *}$ \\
\hline $\begin{array}{l}\text { 10-Tiempo de lectura } \\
\text { de seudopal. (T3) }\end{array}$ & -.011 & $-.592^{* *}$ & $-.429^{* *}$ & $-.516^{* *}$ & -.024 & $-.549^{* *}$ & $.701^{* *}$ & $-.312^{*}$ & $-.527^{* *}$ & 1.000 & $-.317^{*}$ & $-.609^{* *}$ & $.829^{* * *}$ & $-.350^{*}$ & $-.502^{* *}$ & $-.502^{* *}$ \\
\hline $\begin{array}{l}\text { 11-Escritura de pala- } \\
\text { bras (T4) }\end{array}$ & -.172 & $.403^{* *}$ & $.323^{*}$ & $.385^{* *}$ & .068 & $.414^{* *}$ & $-.382^{* *}$ & .246 & $.519^{* *}$ & $-.430^{* *}$ & 1.000 & $.623^{* *}$ & $-.329^{*}$ & $.398^{* *}$ & $.438^{* * *}$ & $.438^{* *}$ \\
\hline $\begin{array}{l}\text { 12-Lectura de pala- } \\
\text { bras (T4) }\end{array}$ & -.140 & $.775^{* *}$ & $.531^{\text {** }}$ & $.708^{* *}$ & .036 & $.780^{* *}$ & $-.466^{* *}$ & $.486^{* *}$ & $.716^{* *}$ & $-.609^{* *}$ & $.609^{* *}$ & 1.000 & $-.510^{* *}$ & $.540^{* *}$ & $.788^{* *}$ & $.788^{* *}$ \\
\hline $\begin{array}{l}\text { 13-Tiempo de lectura } \\
\text { de palabras (T4) }\end{array}$ & .016 & $-.576^{* *}$ & $-.401^{* *}$ & $-.504^{* *}$ & .020 & $-.528^{* *}$ & $.566^{* *}$ & -.246 & $-.578^{* *}$ & $.829^{* *}$ & $-.531^{* *}$ & $-.510^{* * *}$ & 1.000 & -.266 & $-.424^{* *}$ & $-.424^{* *}$ \\
\hline $\begin{array}{l}\text { 14-Escritura de seu- } \\
\text { dopalabras (T4) }\end{array}$ & -.025 & $.601^{* * *}$ & $.577^{* * *}$ & $.565^{* *}$ & .177 & $.516^{* *}$ & -.239 & $.451^{* *}$ & $.516^{* *}$ & $-.350^{*}$ & $.400^{* *}$ & $.540^{* * *}$ & -.266 & 1.000 & $.568^{* * *}$ & $.568^{* * *}$ \\
\hline $\begin{array}{l}\text { 15-Lectura de seudo- } \\
\text { palabras (T4) }\end{array}$ & -.152 & $.756^{* *}$ & $.478^{* * *}$ & $.680^{* *}$ & .087 & $.705^{* *}$ & $-.443^{* *}$ & $.527^{* *}$ & $.567^{* *}$ & $-.502^{* *}$ & $.485^{* *}$ & $.788^{* *}$ & $-.424^{* *}$ & $.568^{* *}$ & 1.000 & $1.000^{* *}$ \\
\hline $\begin{array}{l}\text { 16-Tiempo de lectura } \\
\text { de seudopalabras } \\
\text { (T4) }\end{array}$ & -.152 & $.756^{* *}$ & $.478^{* * *}$ & $.680^{* * *}$ & .087 & $.705^{* *}$ & $-.443^{* *}$ & $.527^{* *}$ & $.567^{* *}$ & $-.502^{* *}$ & $.485^{* *}$ & $.788^{* * *}$ & $-.424^{* *}$ & $.568^{* * *}$ & $1.000^{* * *}$ & 1.000 \\
\hline
\end{tabular}

* La correlación es significante al nivel 05 (bilateral).

** La correlación es significativa al nivel .01 (bilateral). 
escritura y lectura de los tres tiempos de evaluación. Se observó que a fin de primer grado las correlaciones entre las pruebas que incluían palabras y seudopalabras fueron altas $(r=.706 \mathrm{y} p=.001$ para la escritura y $r=.658$ y $p=.001$ para la lectura). Este nivel de correlación sugiere que ambos tipos de ítems se estaban procesando en ese momento mediante el mismo tipo de mecanismos, es decir, accediendo a recursos fonológicos. Al finalizar tercer grado, sin embargo, las correlaciones entre las pruebas que incluían palabras y seudopalabras obtuvieron correlaciones más bajas $(r=.540 \mathrm{y}$ $p=.001$ para la escritura y $r=.538$ y $p=.001$ para la lectura), hecho que sugiere que el procesamiento fonológico implicado en la lectura de seudopalabras comienza a diferenciarse del procesamiento al que acceden los niños en este momento del desarrollo para leer palabras (procesamiento léxico).

Respecto de las correlaciones de las habilidades de lectura y escritura de palabras y seudopalabras con las habilidades predictoras, los resultados señalan que el conocimiento de las letras correlacionó de manera significativa con la mayoría de las habilidades de lectura y escritura en todos los tiempos evaluados; mientras que la medida de conciencia fonológica correlacionó con la escritura de seudopalabras de fin de primero $(r=.544$; $p=.001)$, segundo $(r=.431 ; p=.001)$ y tercer grados $(r=.301 ; p=.005)$. Por otro lado, la fluidez en el trazado de las letras se correlacionó con las medidas de escritura de palabras y correspondencias inconsistentes y dependientes del contexto de fin de los grados segundo y tercero $(r=.354 \mathrm{y}$ $p=.005$ entre la fluidez en el trazado de las letras y la escritura de correspondencias inconsistentes de segundo; $r=.354$ y $p=.005$ entre la fluidez en el trazado de las letras y la escritura de correspondencias dependientes del contexto de segundo; $r=.407$ y $p=.001$ entre la fluidez en el trazado de las letras y la escritura de correspondencias inconsistentes, y $r=.419$ y $p=.001$ entre la fluidez en el trazado de las letras y las dependientes del contexto de tercer grado). Asimismo, la medida de denominación rápida correlacionó de manera significativa con el tiempo de lectura de fin de los grados segundo $(r=.425$ y $p=.001)$ y tercero $(r=.427$ y $p=.001)$ (tabla 3).

Vale aclarar que la falta de correlaciones entre las medidas de escritura de palabras con correspondencias inconsistentes y dependientes del contexto de fin de primer grado y las habilidades predictoras puede deberse a que, habiendo disociado las puntuaciones del total de la prueba y al ser menor el número de palabras en cada categoría (correspondencias inconsistentes y dependientes del contexto) se pierde la fuerza de las correlaciones. El hecho de que la puntuación del total de la prueba de escritura haya obtenido importantes correlaciones con las medidas de las habilidades predictoras da cuenta de este hecho $(r=.555$ y $p=.001$, escritura y conocimiento del nombre de las letras; $r=.472$ y $p=.001$, escritura y conocimiento del sonido de las letras; $r=.597$ y $p=.001$, escritura y conciencia fonológica, y $r=.444$ y $p=.001$, escritura y denominación rápida).

\section{Regresiones}

Con el fin de identificar qué habilidades se constituyeron como predictores de la escritura de palabras con correspondencias dependientes del contexto e inconsistentes y con el aumento de la velocidad en la lectura de palabras, se llevaron a cabo regresiones por pasos sucesivos. Para ello, previamente, se exploró la posibilidad de obtener una medida unificada a partir de las distintas pruebas de conciencia fonológica y de denominación rápida. Se calcularon una serie de correlaciones entre las pruebas. Dado que los resultados señalaron la existencia de correlaciones entre las tres medidas $(r=.478$ y $p=.005$ entre síntesis de sonidos y omisión de sonidos y sílabas; $r=.394 \mathrm{y}$ $p=.005$ entre omisión de sonidos y sílabas y sonidos inicial, y $r=.433$ y $p=.005$ entre reconocimiento de sonido inicial y síntesis de sonidos). Estas se cargaron a un único factor denominado 
Tabla 3

Correlaciones entre las medidas de lectura y escritura de seudopalabras/palabras con correspondencias inconsistentes y correspondencias dependientes del contexto (T2, T3 y T4) y habilidades predictoras (T1)

\begin{tabular}{|c|c|c|c|c|c|c|}
\hline & $\begin{array}{l}\text { Sonido } \\
\text { letra }\end{array}$ & $\begin{array}{c}\text { Nombre } \\
\text { letra }\end{array}$ & $\begin{array}{l}\text { Fluidez en } \\
\text { el trazado }\end{array}$ & $\begin{array}{l}\text { Conciencia } \\
\text { fonológica }\end{array}$ & $\begin{array}{l}\text { Denominación } \\
\text { rápida }\end{array}$ & $\begin{array}{l}\text { Atención } \\
\text { visual }\end{array}$ \\
\hline Escritura de palabras (inc.) (T2) & .237 & .062 & .062 & .154 & .007 & -.120 \\
\hline Escritura de palabras (dep.) (T2) & .1240 & .198 & .061 & .230 & .273 & -.164 \\
\hline Escritura de seudopalabras (T2) & $.536^{* *}$ & $.519^{* *}$ & .213 & $.544^{* *}$ & $.432^{* *}$ & .229 \\
\hline Lectura de palabras (T2) & $.442^{* *}$ & $.514^{* *}$ & .201 & .218 & $.392^{* *}$ & .206 \\
\hline Lectura de seudopalabras (T2) & $.317^{*}$ & $.387^{* *}$ & .291 & .237 & $.300^{*}$ & .199 \\
\hline Escritura de palabras (inc.) (T3) & .219 & .134 & $.354^{*}$ & .078 & .124 & -.064 \\
\hline Escritura de palabras (dep.) (T3) & .187 & .047 & $.354^{*}$ & .184 & .053 & -.180 \\
\hline Escritura de seudopalabras (T3) & $.447^{* *}$ & $.391^{* *}$ & .096 & $.431^{* *}$ & .208 & $.270^{* *}$ \\
\hline Lectura de palabras (precisión) (T3) & $.393^{* *}$ & $.400^{* *}$ & .254 & .172 & $.515^{* *}$ & .215 \\
\hline Lectura de palabras (tiempo) (T3) & -.247 & .233 & .005 & .046 & $.467^{*}$ & .067 \\
\hline Lectura de seudopalabras (precisión) (T3) & $.437^{* *}$ & $.447^{* *}$ & .013 & .255 & .273 & .125 \\
\hline Lectura de seudopalabras (tiempo) (T3) & -.238 & -.228 & .144 & .051 & $.425^{* *}$ & .063 \\
\hline Escritura de palabras (inc.) (T4) & .232 & $.425^{* *}$ & $.407^{* *}$ & .047 & $-.431^{*}$ & .211 \\
\hline Escritura de palabras (dep.) (T4) & .253 & $.363^{*}$ & $.419^{* *}$ & .271 & $.394^{* *}$ & .056 \\
\hline Escritura de seudopalabras (T4) & .371 & $.445^{* *}$ & .043 & $.301^{*}$ & $.335^{*}$ & .043 \\
\hline Lectura de palabras (precisión) (T4) & .255 & $.399^{* *}$ & .218 & .175 & $.317^{*}$ & .297 \\
\hline Lectura de palabras (tiempo) (T4) & -.291 & $.300^{*}$ & .118 & .053 & $.427^{* *}$ & .046 \\
\hline Lectura de seudopalabras (precisión) (T4) & .184 & .285 & .142 & .051 & .206 & $.297^{*}$ \\
\hline Lectura de seudopalabras (tiempo) (T4) & .184 & .285 & .141 & .051 & .207 & $.297^{*}$ \\
\hline
\end{tabular}

conciencia fonológica que en el análisis exploratorio explicó un $55.81 \%$ de la variancia total de las pruebas. En el caso de las pruebas de denominación rápida, dadas también las altas correlaciones existentes entre las tres medidas $(r=.689$ y $p=.001$ entre la prueba tradicional y la prueba de dígitos; $r=.795$ y $p=.001$ entre la prueba de dígitos y la prueba alternativa, y $r=.783, p=.001$ entre la prueba alternativa y la prueba tradicional), se las cargó a un único factor denominado denominación rápida que en el análisis exploratorio explicó un $84.05 \%$ de la variancia total de las pruebas. En la tabla 4 se expresan la carga factorial y las comunalidades de los factores, denominados conciencia fonológica y denominación rápida.
Tabla 4

Carga factorial y comunalidades de los factores denominado conciencia fonológica y denominación rápida

\begin{tabular}{lcc}
\hline & Factor & H2 \\
\hline Omisión de sílabas & .81 & .65 \\
Síntesis de sonidos & .82 & .66 \\
$\begin{array}{l}\text { Reconocimiento de sonido } \\
\text { inicial }\end{array}$ & .63 & .39 \\
$\begin{array}{l}\text { Denominación rápida de letras } \\
\text { (con dificultad fonológica) }\end{array}$ & .93 & .86 \\
$\begin{array}{l}\text { Denominación rápida de letras } \\
\begin{array}{l}\text { Denominación rápida de } \\
\text { dígitos }\end{array}\end{array}$ & .94 & .88 \\
\hline
\end{tabular}


En cada una de las regresiones se introdujo una de las pruebas de escritura (de correspondencias inconsistentes o dependientes del contexto) y precisión y tiempo de lectura de palabras de los grados segundo y tercero como variable dependiente (salvo a fin de primer grado, que se introdujo en la medida de escritura sin disociar entre tipos de correspondencias por haberse perdido las correlaciones). Las variables independientes introducidas fueron las habilidades de denominación rápida, conciencia fonológica, fluidez en el trazado de las letras, conocimiento de las letras y atención visual, administradas a principio de primer grado, por lo que se obtuvieron predictores longitudinales de las medidas de aprendizaje ortográfico de fin de segundo y tercero (tabla 5).

Los resultados de las regresiones mostraron que la medida de fluidez del trazado de las letras predijo la escritura convencional de palabras con correspondencias inconsistentes de fin los grados segundo y tercero y la escritura de correspondencias dependientes del contexto de fin de segundo grado. La escritura de correspondencias dependientes del contexto de fin de tercero tuvo como mejor predictor al conocimiento del nombre de las letras.

La precisión lectora de fin de segundo y tercer grados tuvo como mejor predictor el conocimiento del nombre de las letras de principio de primer grado. Mientras que la velocidad en el reconocimiento tuvo como mejor predictor a las medidas de denominación rápida.

\section{Discusión}

El presente estudio se propuso analizar la interacción entre procesos fonológicos y ortográficos en momentos iniciales del aprendizaje de la lectura y la escritura por parte de niños hablantes de español. Para ello, el presente estudio también se propuso analizar cuáles son las habilidades que predicen la escritura ortográficamente convencional de palabras con correspondencias inconsistentes en español.
Tabla 5

Resumen de las habilidades predictoras (longitudinales) de la lectura y de la escritura de palabras con correspondencias inconsistentes y correspondencias dependientes del contexto fin de los grados primero, segundo y tercero

\begin{tabular}{|c|c|c|c|c|c|}
\hline $\begin{array}{c}\text { Variable } \\
\text { dependiente }\end{array}$ & $\begin{array}{l}\text { Variables } \\
\text { predictoras }\end{array}$ & $\mathrm{r}$ & $r^{2}$ & $\mathrm{~F}$ & Sig. \\
\hline \multirow{2}{*}{$\begin{array}{l}\text { Escritura de pa- } \\
\text { labras fin primer } \\
\text { grado }\end{array}$} & Nombre letra & .597 & .357 & 24.4 & .001 \\
\hline & $\begin{array}{l}\text { Conciencia } \\
\text { fonológica }\end{array}$ & .653 & .427 & 15.9 & .001 \\
\hline \multirow[t]{2}{*}{$\begin{array}{l}\text { Precisión en la lec- } \\
\text { tura de palabras fin } \\
\text { de primer grado }\end{array}$} & $\begin{array}{l}\text { Nombre } \\
\text { Letra }\end{array}$ & .561 & .315 & 20.2 & .001 \\
\hline & Sonido letra & .617 & .381 & 13.2 & .001 \\
\hline $\begin{array}{l}\text { Escritura de pala- } \\
\text { bras fin segundo } \\
\text { grado (corres. in.) }\end{array}$ & $\begin{array}{l}\text { Fluidez en } \\
\text { el trazado de } \\
\text { las letras }\end{array}$ & .364 & .133 & 6.7 & .013 \\
\hline $\begin{array}{l}\text { Escritura de pala- } \\
\text { bras fin segundo } \\
\text { grado (corres. dep.) }\end{array}$ & $\begin{array}{l}\text { Fluidez en } \\
\text { el trazado de } \\
\text { las letras }\end{array}$ & .334 & .111 & 5.4 & .023 \\
\hline $\begin{array}{l}\text { Precisión en la lec- } \\
\text { tura de palabras fin } \\
\text { segundo grado }\end{array}$ & $\begin{array}{l}\text { Denomina- } \\
\text { ción rápida }\end{array}$ & .437 & .191 & 10.4 & .002 \\
\hline $\begin{array}{l}\text { Tiempo de lectura } \\
\text { de palabras de se- } \\
\text { gundo grado }\end{array}$ & $\begin{array}{l}\text { Denomina- } \\
\text { ción rápida }\end{array}$ & .300 & .093 & 4.2 & .027 \\
\hline $\begin{array}{l}\text { Escritura de pala- } \\
\text { bras fin de tercer } \\
\text { grado (corres. inc.) }\end{array}$ & $\begin{array}{l}\text { Fluidez en } \\
\text { el trazado de } \\
\text { las letras }\end{array}$ & .322 & .295 & 5.0 & .029 \\
\hline $\begin{array}{l}\text { Escritura de pala- } \\
\text { bras fin de tercer } \\
\text { grado (corres. dep.) }\end{array}$ & Nombre letra & .487 & .237 & 13.6 & .001 \\
\hline $\begin{array}{l}\text { Precisión en la lec- } \\
\text { tura de palabras fin } \\
\text { tercer grado }\end{array}$ & Nombre letra & .301 & .091 & 4.4 & .042 \\
\hline $\begin{array}{l}\text { Tiempo de lectura } \\
\text { de palabras de ter- } \\
\text { cer grado }\end{array}$ & $\begin{array}{l}\text { Denomina- } \\
\text { ción rápida }\end{array}$ & .427 & .182 & 9.7 & .003 \\
\hline
\end{tabular}

A fin de primer grado los sujetos escribieron correctamente el $40 \%$ de las palabras con correspondencias inconsistentes (medida de conocimiento 
ortográfico); mientras que la lectura - tarea que los sujetos pueden resolver a partir del procesamiento fonológico y de su conocimiento de las correspondencias - fue del 75\%. Estos resultados coinciden con otros obtenidos en lenguas de ortografía transparente, los cuales indican que en momentos iniciales del aprendizaje los niños llevan a cabo tareas de lectura y escritura accediendo al procesamiento fonológico (Sánchez et al., 2009; Share, 2004). El hecho de que la prueba de escritura de palabras y seudopalabras haya estado altamente correlacionada en este momento y de que la prueba de escritura haya tenido una importante correlación con el nivel de conciencia fonológica de los sujetos constituye evidencia extra de esta relación.

Por otra parte, el alto desempeño en lectura de palabras $(75 \%)$ y de seudopalabras $(78 \%)$ y en escritura de seudopalabras $(65 \%)$ constituye también un indicador del dominio de los mecanismos fonológicos alcanzado por los niños. En el presente estudio, los predictores de las habilidades de lectura y escritura dan apoyo a la noción de la primacía de mecanismos fonológicos. Así, los resultados de las regresiones mostraron que la medida de conciencia fonológica predijo la escritura a fin de primer grado; entre tanto, el conocimiento de las letras resultó el mejor predictor de la lectura. Así, conocimiento de letras y conciencia fonológica constituyen los pilares del desarrollo de la vía fonológica de procesamiento de palabras (Plaut, McClelland, Seidenberg \& Patterson, 1996).

Cabe señalar que el hecho de que la conciencia fonológica haya sido el mejor predictor de la escritura, pero no de la lectura, sugiere que, en lenguas de ortografía transparente, la escritura demanda mayores recursos de procesamiento fonológicos que la lectura de palabras (Babayig it \& Stainthorp, 2007). Estos resultados parecen señalar que la conciencia fonológica posee diferente nivel de impacto en la lectura y la escritura, por lo que esta última es una medida más sensible a las tareas de procesamiento fonológico que la primera (Bosman \& van Orden, 1997; Perfetti, 1997).
Ahora bien, se obtuvo también un resultado que parecería contradictorio con la idea del predominio inicial del procesamiento fonológico: desde fines de primer grado se encontró un efecto de frecuencia de las palabras tanto para la lectura como para la escritura. Este efecto es considerado un indicador del desarrollo de mecanismos léxicos de procesamiento. Es posible que este efecto se relacione con el hecho de que en la prueba de lectura y escritura de palabras ortográficamente complejas se incluyeron palabras con frecuencias extremas, es decir, muy altas o muy bajas. La inclusión de palabras altamente familiares para los niños haría que, ya desde fines de primer grado, los niños hayan tenido representaciones ortográficas de algunas palabras a pesar de que los resultados concluyen que en esta etapa predominan las estrategias fonológicas de lectura y escritura. En cualquier caso, se trata de un resultado que, en coincidencia con estudios previos, sugiere que la interacción entre fonología y ortografía es compleja, ya desde el inicio del aprendizaje (Sánchez et al., 2009) siendo el mecanismo de recodificación fonológica el encargado de realizar el paso de no familiar a familiar de cada ítem léxico (Share, 2008).

Los resultados de fines de segundo grado señalan ya un creciente dominio de recursos de origen léxico. Efectivamente, la escritura de palabras con correspondencias inconsistentes había ascendido al 58\% y los niños leían en promedio una palabra por segundo; pero les tomaba 5 segundos leer cada seudopalabra. Si bien la precisión lectora resultó muy similar para ambos tipos de estímulo $(88 \%$ de las seudopalabras y $93 \%$ de las palabras fueron correctamente leídas), el tiempo de lectura mostró importantes diferencias. En este sentido, se ha señalado que, dado que las características de las lenguas de ortografía transparente determinan que el logro de la precisión lectora se alcance tempranamente, las diferencias individuales se trasladan a la velocidad (Share, 2008; Wimmer, 2006). El efecto de lexicalidad encontrado en relación con el tiempo de lectura, unido al efecto de frecuencia verificado en 
la lectura de palabras, sugiere que el proceso lector se encuentra a partir de segundo grado, cada vez más apoyado en el procesamiento léxico. En este sentido, y en coincidencia con numerosos trabajos previos, el mejor predictor de las medidas de lectura (tanto de la precisión como de la velocidad) no fue la conciencia fonológica ni el conocimiento de las letras, sino la prueba de denominación rápida (Furnes \& Samuelsson, 2009; Landerl \& Wimmer, 2008; Wolf, Bowers \& Biddle, 2000). Se considera que la tarea de denominación rápida se relaciona con la facilidad con que lo sujetos establecen y recuperan asociaciones entre una forma gráfica y su correspondiente etiqueta fonológica (Berninger et al., 2001; Manis, Seidenberg \& Doi, 1999).

Resultados similares se obtuvieron a fines de tercer grado momento en el que siguió mejorando la escritura de palabras con correspondencias inconsistentes y el tiempo de reconocimiento de las palabras, lo cual sugiere nuevamente, al igual que a fin de segundo grado, un incremento del almacén de representaciones ortográficas.

Ahora bien, tanto a fin de segundo como de tercer grado, el desempeño en la prueba de escritura de palabras ortográficamente complejas, tanto con correspondencias dependientes del contexto como inconsistentes, fue significativamente inferior al obtenido para la lectura. Este hecho parecería contradecir la noción de que los niños cuentan con representaciones ortográficas de las palabras. En efecto, la velocidad alcanzada en la lectura sugeriría la presencia de representaciones ortográficas. Pero dado que las representaciones ortográficas de las palabras son las que indican la escritura exacta de ellas, el hecho de que los niños hayan obtenido una puntuación relativamente baja en la escritura convencional de palabras ortográficamente complejas sugeriría la inexistencia de representaciones de cada ítem léxico. Sin embargo, las diferencias observadas entre el desempeño en la lectura y en la escritura pueden interpretarse en el marco propuesto por Perfetti (1992). Señala el autor que existirían diferencias en términos de calidad en las represen- taciones ortográficas de las palabras. El modelo contempla el desarrollo de un léxico funcional en el cual las representaciones poseen características no especificadas en su totalidad y de un léxico autónomo, en el cual se incluyen representaciones completamente especificadas. El tránsito del léxico funcional al autónomo se relaciona con el acceso a diferentes fuentes de información que interactúan (asociaciones letras-fonemas, patrones ortográficos) y se vuelven redundantes, lo cual fortalece las representaciones.

Es posible pensar que en una lengua de ortografía transparente las representaciones del léxico funcional resultarían suficientes para la lectura precisa y veloz del ítem, pero insuficientes para su correcta representación ortográfica. En consecuencia, se puede pensar que, en el presente estudio, la ventaja de las palabras sobre las seudopalabras se relaciona con que, desde fines de segundo grado un número considerable de palabras estarían incorporadas al léxico funcional, lo que daría lugar a una lectura veloz. No obstante, la incorporación de una palabra ortográficamente compleja al léxico funcional no resultaría suficiente para su representación escrita convencional, por lo que solamente fueron correctamente escritas aquellas palabras que alcanzaban un estatus autónomo. De ahí la diferencia entre los puntajes de lectura y escritura.

La idea de que el estatus autónomo se logra a partir de la redundancia en las fuentes de información podría dar cuenta de que en el presente estudio la prueba de fluidez en el trazado de las letras fue el mejor predictor de la escritura de fin de los grados segundo y tercero. Es posible pensar que la práctica de la escritura manuscrita, así como otras formas de exploración táctil de las letras, contribuye a formar conexiones entre la forma fonológica y su forma gráfica (haptic bond effect) (Bara et al., 2004, 2007; van Hell, Bosman $\&$ Bartelings, 2003). Este efecto se debería a que tanto la escritura como otras tareas que involucran actividades táctiles requieren el procesamiento secuencial de los elementos que componen la palabra 
en un examen activo e intencional de cada uno de los estímulos que se presentan en orden fijo y de manera lineal. Esta forma de procesamiento promovería que se destinen recursos atencionales a la localización, el tamaño y la forma de las letras en un proceso mucho menos global y más analítico que en la lectura (Bara et al., 2004, 2007; Berger \& Hatwell, 1993, 1996).

Es posible pensar que este tipo de procesamiento contribuiría a formar el tipo de representación autónoma que da lugar a la escritura ortográficamente convencional; de ahí el papel predictor del trazado de las letras sobre este conocimiento. Como se dijo, varios estudios han aportado evidencia de que la tarea de escribir las palabras (más allá de la calidad caligráfica del output) generaría un link adicional entre las formas fonológicas y las formas ortográficas, lo cual facilitaría la formación de representaciones ortográficas completamente especificadas de las palabras (Berninger et al., 1992; Cunningham \& Stanovich, 1991).

Por último, vale detenerse en los resultados obtenidos en relación con la escritura de diferentes tipos de correspondencias (inconsistentes y dependientes del contexto). Como se señaló, la escritura de correspondencias dependientes del contexto se asocia con el conocimiento de reglas de correspondencia fonema-grafema; mientras que la escritura convencional de palabras con correspondencias inconsistentes no responde a ninguna regla específica. Con esta diferencia es posible pensar en la existencia de una brecha importante entre la adquisición de ambos tipos de dificultad, pues resulta en una mayor facilidad la adquisición de reglas. Sin embargo, los datos obtenidos en el presente estudio sugieren que el conocimiento sobre ambos tipos de correspondencias se produce de manera bastante simultánea, ya que recién a fin de tercer grado se obtuvieron diferencias estadísticamente significativas a favor del conocimiento de reglas contextuales. En este sentido, cabría preguntarse si los niños en edad escolar adquieren reglas de correspondencias complejas o si lo que se produce es el almacenamiento de material subléxico (en este caso de sílabas), que permite la correcta escritura de palabras que contienen las sílabas almacenadas.

En términos generales, los resultados obtenidos en el presente estudio coinciden con otros que han planteado que en una lengua de ortografía trasparente prima, en momentos iniciales, el procesamiento fonológico para la escritura y la lectura de palabras (Sánchez et al., 2009; Signorini, 1997; Share, 2004); pero que a medida que se automatiza el mecanismo de recodificación fonológica, comienza a incrementarse significativamente el almacén léxico de los sujetos. Los resultados obtenidos también señalan la importancia de las habilidades de denominación rápida y de tareas relacionadas al desarrollo de patrones grafomotores en la formación de representaciones ortográficas de las palabras.

\section{Referencias}

Babayiğit, S. \& Stainthorp, R. (2007). Preliterate phonological awareness and early literacy skills in Turkish. Journal of Research in Reading, 30(4), 394-413. doi: 10.1111/j.14679817.2007.00350.x

Bara, F., Gentaz, E., \& Colé, P. (2007). Haptics in learning to read with children from low socioeconomic status families. British Journal of Developmental Psychology, 25(4), 643-663. doi: 10.1348/026151007X186643

Bara, F., Gentaz, E., Sprenger-Charolles, L., \& Colé, P. (2004). The visuo-haptic and haptic exploration of letters increases the kindergarten-children's reading acquisition. Cognitive Development, 19, 433-449.

Berger, C. \& Hatwell, Y. (1993). Dimensional and overall similarity classifications in haptics: A developmental study. Cognitive Development, 8 , 495-516. doi: 10.1016/S0885-2014(05)80006-1

Berger, C., \& Hatwell, Y. (1996). Developmental trends in haptic and visual free classifications: Influence of stimulus structure and exploration 
on decisional processes. Journal of Experimental Child Psychology, 63, 447-465.

Berninger, V., Abbott, R., Thomson, J., \& Raskind, W. (2001). Language phenotype for reading and writing disability: A family approach. Scientific Studies in Reading, 5, 59-105. doi: 10.1177/0022219410391189

Berninger, V. \& Graham, S. (1997). Treatment of handwriting problems in beginning writers: Transfer from handwriting to composition. Journal of Educational Psychology, 89, 652666. doi: 10.1037/0022-0663.89.4.652

Berninger, V., Yates, C., Cartwright, A., Rutberg, J., Remy, E., \& Abbott, R. (1992). Lower-level developmental skills in beginning writing. Reading and Writing: An Interdisciplinary Journal, 4, 257-280. doi: 10.1177/0741088311410188

Bosman, A. M. T., \& Van Orden, G. C. (1997). Why spelling is more difficult than reading. In C. A. Perfetti, L. Rieben \& M. Fayol (Eds.), Learning to spell (pp. 173-194). Hillsdale, NJ: Lawrence Erlbaum Associates.

Bosse, M., Tainturier, M., \& Valdois, S., (2007). Developmental dyslexia: The visual attention span deficit hypothesis. Cognition, 104, 198-230. doi:10.1016/j.cognition.2006.05.009

Bourke, L. \& Adams, A. M. (2010), Cognitive constraints and the early learning goals in writing. Journal of Research in Reading, 33, 94-110. doi: 10.1111/j.1467-9817.2009.01434.x

Cardoso-Martins, C. \& Corrêa, M. F. (2008). O desenvolvimento da escrita nos anos pré-escolares: Questões acerca do estágio silábico [The development of spelling skills in the preschool years: questions about the syllabic stage]. Psicologia: Teoria e Pesquisa, 24, 279-286. doi: http://dx. doi.org/10.1590/S0102-37722008000300003

Compton, D. (2003). The influence of item composition on RAN: Letter performance in first-grade children. The Journal of Special Education, 37(2), 81-94.

Cunningham, A. E. \& Stanovich, K. E. (1991). Tracking the unique effects of print exposu- re in children: Associations with vocabulary, general knowledge, and spelling. Journal of Educational Psychology, 83, 264-274. doi: 10.1037/0022-0663.83.2.264

Defior Citoler, S., Fonseca, L., Gottheil, B., Aldrey, A., Jiménez Fernández, G., Pujals, M., Rosa, G., $\&$ Serrano Chica, F. D. (2006). Test de lectura y escritura en español [Reading and writing test in Spanish]. Buenos Aires: Paidós.

Denckla, M. B. \& Rudel, R. (1976). Rapid automatized naming (RAN): Dyslexia differentiated from other learning disabilities. Neuropsychologia, 14(4), 471-479. doi: 10.1016/00283932(76)90075-0

Diuk, B., Borzone, A. M., Sánchez Abchi, V., \& Ferroni, M. (2009). La adquisición de conocimiento ortográfico en niños de $1^{\circ}$ a $3^{\circ}$ año de educación básica [Orthographic knowledge acquisition in $1^{\text {st }}$ to $3^{\text {rd }}$ year of primary school]. Psyché, 18(1), 61-71. doi: 10.4067/S071822282009000100006

Ehri, L. C. (1992). Reconceptualizing the development of sight word reading and its relationship to recoding. En P. B. Gough, L. C. Ehri y R. Treiman (Eds.), Reading acquisition. Hillsdale, NJ: Erlbaum.

Ferreres, A., Martínez Cuitiño, M., Jacubovich, S., Olmedo, A. \& López, C. (2003). Las alexias y los modelos de doble ruta de lectura en hispanohablantes. Revista Argentina de Neuropsicología, 1, 37-52.

Furnes, B. \& Samuelsson, S. (2009). Preschool cognitive and language skills predicting kindergarten and grade 1 reading and spelling: A cross-linguistic comparison. Journal of Research in Reading, 32(3), 275-292. doi: 10.1111/j.14679817.2009.01393.x

Hernández Sampieri, R., Fernández Collado, C., \& Baptista Lucio, P. (2006). Metodología de la investigación. México: Mc Graw Hill.

Jiménez Gonzáles, J. E. \& Ortiz Gonzáles M. R. (1995). Conciencia fonológica y aprendizaje de la lectura: teoría, evaluación e intervención 
[Phonological awareness and reading acquisition: theory, assessment and intervention]. Madrid: Síntesis.

Jiménez, J. E., O'Shanahan, I., Tabraue el Jaber, M., Artiles, C., Muñetón, M., Guzmán, R., Naranjo. F. \& Rojas, E. (2008). Evolución de la escritura de palabras de ortografía arbitraria en lengua española [Spelling development in the Spanish language]. Psicothema, 20(4), 786-794.

Landerl, K., \& Wimmer, H. (2008). Development of word reading fluency and spelling in a consistent orthography: An 8-year follow-up. Journal of Educational Psychology, 100(1), 150-161. doi: 10.1037/0022-0663.100.1.150

Maisto, A. \& Morris. C. (2001). Introducción a la psicología. México: Prentice Hall.

Manis, F. R., Seidenberg, M. S., \& Doi, L. M. (1999). See dick RAN: Rapid naming and the longitudinal prediction of reading sub skills in first and second grades. Scientific Studies of Reading, 3, 129-157. doi: 10.1177/002221940203500306

Martínez Martín, J. A., \& García Pérez, E. (2004). Diccionario de frecuencias del castellano escrito en niños de 6 a 12 años [Frequency dictionary of Spanish in children 6 to 12 years]. Salamanca: Universidad Pontificia de Salamanca.

Mattingly, I. G. (1972). Reading, the linguistic process, and linguistic awareness. In J. F. Kavenagh \& I. G. Mattingly (Eds.), Language by ear and by eye. Cambridge, Mass: MIT Press.

McCrory, E., Mechelli, A., Frith, U., \& Price, C. (2005). More than words: A common neural basis for reading and naming deficits in developmental dyslexia? Brain, 128, 261-267. doi: 10.1093/brain/awh340

Perfetti, C.A. (1992). The representation problem in reading acquisition. En P.B. Gough, L.C. Ehri y R. Treiman (Eds.), Reading acquisition (pp. 145-174). Hillsdale, NJ: Erlbaum.

Perfetti, C.A. (1997). The psycholinguistics of spelling and reading. In C.A. Perfetti, L. Rieben, \& M. Fayol (Eds.). Learning to spell: Research, theory, and practice across languages (pp. 2138). Mahwah, NJ: Erlbaum.

Plaut, D., McClelland, J., Seidenberg, M., \& Patterson, K (1996). Understanding normal and impaired word reading: Computational principles in Quasi-regular domains. Psychological Review, 103, 56-115. doi: 10.1037/0033295X.103.1.56

Plaza, M. \& Cohen, H. (2006). The contribution of phonological awareness and visual attention in early reading and spelling. Dyslexia, 13(1), 67-76. doi: 10.1002/dys.330

Salles, J. F. \& Parente, M. (2006). Heterogeneidade nas estratégias de leitura/escrita em crianças com dificuldades de leitura e escrita [Heterogeneity in the reading/writing strategies in children with reading and writing difficulties]. Psico, 37(1), 83-90.

Sánchez Abchi, V., Borzone, A.M., \& Diuk, B. (2007). La escritura de textos en niños pequeños: relación entre la trascripción y la composición [Text writing in small children: relationship between transcription and composition]. Universitas Psychologica, 6(3), 559-569.

Sánchez Abchi, V., Diuk, B., Borzone, A., \& Ferroni, M. (2009). La escritura de palabras en la alfabetización inicial: interacción entre el conocimiento fonológico y ortográfico [Spelling Development in Spanish: Interaction between phonological and orthographic knowledge]. Interdisciplinaria, 26(1), 95-119.

Savage, R., Pillay, V., \& Melidona, S. (2008). Rapid serial naming is a unique predictor of spelling in children. Journal of Learning Disabilities, 41,235-250. doi: 10.1177/0022219408315814 Shahar-Yames, D. \& Share, D. L. (2008). Spelling as a self-teaching mechanism in orthographic learning. Journal of Research in Reading, 31, 22-39. doi: 10.1111/j.1467-9817.2007.00359.x

Share, D. L. (1995). Phonological recoding and self-teaching: Sine qua non of reading acquisition. Cognition, 55, 151-218. doi:10.1016/00100277(94)00645-2 
Share, D. L. (2004). Orthographic learning at a glance: On the time course and developmental onset of self-teaching. Journal of Experimental Child Psychology, 87, 267-289. doi: 10.1016/j. jecp.2004.01.001

Share, D. L. (2008). On the Anglocentricities of current reading research and practice: The perils of over-reliance on "outlier" orthography. Psychological Bulletin, 134, 584-616. doi: 10.1037/0033-2909.134.4.584

Share, D. L. (2011). On the role of phonology in reading acquisition: The self-teaching hypothesis. En S. A. Brady, D. Braze \& C. A. Fowler (Eds.), Explaining individual differences in reading: Theory and evidence. New directions in communication disorders research (pp. 45-68). New York, NY: Psychology Press.

Share, D., \& Shalev, C. (2004). Self-teaching in normal and disable children. Reading and Writing, 17, 769-800. doi: 10.1037/a0019038

Signorini, A. (1997). Word reading in Spanish: A comparison between skilled and less skilled beginning readers. Applied Psycholinguistics, 18, 319-344. doi: http://dx.doi.org/10.1017/ S014271640001050X

Signorini, A., Borzone, A. M. \& Diuk, B. (2001). Del conocimiento fonológico al conocimiento ortográfico. Alcance y limitaciones de la perspectiva "fonocéntrica" en el procesamiento de palabras escritas. Lenguas Modernas, 28, 7-28.

Signorini, A. \& Piacente, T. (2001). Adquisición de la lectura en español: Las habilidades de procesamiento de palabras en lectores iniciales [Acqui- sition of reading in Spanish: word processing skills in early readers]. Revista Irice, 15, 5-29. Treiman, R. \& Bourassa, D. (2000). Children's written and oral spelling. Applied Phsycholinguistics, 21, 183- 204. doi: 10.1093/wsr/wsq001

van Hell, J. G., Bosman, A. M. T., \& Bartelings, M. (2003). Visual dictation improves the spelling performance of three groups of Dutch children with spelling disabilities. Learning Disability Quarterly, 26, 239-255. doi: 10.2307/1593637 Wimmer, H. (2006). Don't neglect reading fluency! Developmental Science, 9(5), 447-448. doi: 10.1111/j.1467-7687.2006.00527.x

Wimmer, H., \& Mayringer, H. (2002). Dysfluent reading in the absence of spelling difficulties: A specific disability in regular orthographies. Journal of Educational Psychology, 94, 272277. doi: 10.1037/0022-0663.94.2.272

Wolf, M., Bowers, P., \& Biddle, K. (2000). Naming-speed processes, timing, and reading: A conceptual review. Journal of Learning Disabilities, 33, 387-407. doi: 10.1177/0022219 40003300409

\section{Anexo. Prueba de atención visual administrada en el T1 y T2}

\begin{tabular}{|c|c|c|c|c|c|c|c|c|c|}
\hline 75 & $\not S$ & $Q$ & \& & 8 & Is & $\not 5$ & $\&$ & d8 & $Q$ \\
\hline$\&$ & $Q$ & $\not s$ & 88 & $Q$ & 85 & Is & 28 & $\mathscr{S}$ & 15 \\
\hline S & $\not 5$ & $\mathscr{D}$ & $\not$ & $\mathscr{D}$ & $Q$ & d & 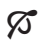 & b & \\
\hline$Q$ & \& & 8 & $\alpha$ & $\not 5$ & $\infty$ & 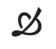 & $a$ & $\varnothing$ & 7 \\
\hline \& & $\not S$ & $Q$ & $d 8$ & 75 & Q & 85 & $\not s$ & \& & \\
\hline
\end{tabular}

Fecha de recepción: 14 de octubre de 2014 Fecha de aceptación: 15 septiembre de 2015 
